\title{
An Enhanced Approach for Automation the Diagnosis of Iron Deficiency Anemia Based on Quantitative Analysis of Red Blood Cells in Intestine Villi Tissue
}

\author{
Manar Rizik Al-Sayyed ${ }^{1}$, Faten Hamad ${ }^{2}$, Rizik Al-Sayyed ${ }^{3}$ \& Hussam N. Fakhouri ${ }^{3}$ \\ ${ }^{1}$ Histopathology and Cytology Section, Department of Medical Laboratory and Forensic Medicine, Jordan \\ University Hospital, Amman, Jordan \\ ${ }^{2}$ School of Educational Sciences, The University of Jordan, Amman, Jordan \\ ${ }^{3}$ King Abdullah II School of Information Technology, The University of Jordan, Amman, Jordan \\ Correspondence: Manar Rizik Al-Sayyed, Jordan University Hospital, Amman, Jordan. E-mail: \\ dr.manar216@gmail.com
}

Received: Oct. 9, 2018

Accepted: Oct. 17, 2018

Online Published: Nov. 16, 2018

doi:10.5539/mas.v12n12p65

URL: https://doi.org/10.5539/mas.v12n12p65

\begin{abstract}
Recent years have witnessed a huge revolution in developing automated diagnosis for different diseases such as cancer using medical image processing. Many researchers have been conducted in this field. Analyzing medical microscopic images provide pathology medical track with large information about the status of the patients and the progress of the diseases and help in detecting any pathological changes in tissues. Automation of the diagnosis of these images will lead to a better, faster and enhanced diagnosis for different hematological and histological images. This paper proposes an automated approach for analyzing blood smear microscopic images to help in diagnosing anemia using quantitative analysis of red blood cells in intestine villi tissue. The diagnoses depends on counting the number of blue and red stained blood cells that contain iron in each villi separately, then, it calculates the percentage of blue cells and red cells in the experimented image. The experimental results have shown that using digital image processing techniques through processing the image into different stages as including noise removal, image sharpening, enhancing contrast, find region of interest, isolating color, removing edges, and counting cells leads to a successful outcome and the diagnose of anemia.
\end{abstract}

Keywords: anemia, blood deficiency, image processing, RBC, anemia diagnosis

\section{Introduction}

Anemia is a group of clinical and hematological syndrome, where is the hemoglobin concentration in the blood is decreased (Chard, 1990), (Janz, Johnson, \& Rubenstein, 2013).It is also a lowered ability of the blood to carry oxygen, more often with a simultaneous decrease in the number of erythrocytes (or general the volume of erythrocytes). The term "anemia" without specification does not define a specific disease, that is, anemia should be considered one of the symptoms of various pathological conditions. It is necessary to distinguish between hydremia (pseudoanemia, for example, pregnant women) and anemia, where with hydremia the number of erythrocytes and hemoglobin remains the same, but the volume of the liquid part of the blood increases (Janz, Johnson, \& Rubenstein, 2013).

The reduction of hemoglobin concentration in the blood often occurs with a simultaneous decrease in the number of red blood cells and a change in their qualitative composition (Kumar, Abbas, \& Aster, 2017). Any anemia leads to a decrease in the respiratory function of the blood and the development of oxygen starvation of tissues, which is most often expressed by symptoms such as pale skin, fatigue, weakness, headaches, dizziness, palpitations, shortness of breath, and others(Calbet, Lundby, Koskolou, \& Boushel, 2006).Anemia can occur as a symptom of variety of diseases that can either be related to the primary damage to the blood system or independent of it. In this regard, a strict nosological classification of anemia is impossible. To classify anemia, it is customary to use the principle of practical expediency (Smith, 2009), (Janz, Johnson, \& Rubenstein, 2013).

In a routine study of the smear of peripheral blood, the morphologist indicates a deviation of the size of red blood cells to a smaller side (microcytosis) or to a greater side (macrocytosis), but such an estimate, if produced without 
special adaptations - micrometers, cannot be free from subjectivism. The advantage of automatic blood analysis is the standardization of the indicator - the average corpuscular volume (RMS), measured in femtolitra (fl). The RMS is directly measured using an automated meter. The normal value of the average corpuscular volume is $80-90 \mathrm{fl}$ (Normocytosis). The increase of RMS above $95 \mathrm{fl}$ macrocytosis, while the decrease of RMSto be less than $80 \mathrm{fl}$ microcytosis. However, the disadvantage is the relatively expensive and complex equipment that requires appropriate maintenance (Beutler, \& West, 2005), (Beutler, \& Waalen, 2006). (Janz, Johnson, \& Rubenstein, 2013).

Anemia is diagnosed by blood count - a blood test. It can be caused by the destruction of red blood cells (haemolysis) or blood loss (haemorrhage), which is called peripheral or regenerative anemia. it can also be due to a decrease in the production of hemoglobin, in this case it is called central or non-regenerative anemia.If the decrease concerns the red blood cells and also the thrombocytes or the leucocytes, one speaks then of bicytopenia(Beutler, \& Waalen, 2006).(Janz, Johnson, \& Rubenstein, 2013).

\subsection{Common Signs and Symptoms of Anemia}

First of all, cutaneous pallor (which is not always obvious and which the patient and his family get used to if anemia sets in very gradually). The paleness of the mucous membranes and especially conjunctiva is another symptom that is more obvious (Ludwig \& Strasser, 2001), (Kumar, Abbas, \& Aster, 2017). Observation of the inside of the lower eyelid is also used to detect anemia. In animals covered with feathers, hairs or scales, anemia is visible only on the visible mucosa; tachycardia, which allows, at the beginning, to maintain a correct oxygenation of the tissues when the hemoglobin is low by increasing the cardiac output. Thisoccurs first at the effort (climbing stairs) then it becomes permanent at rest; dyspnea with polypnea has the same role. The neuro-sensory signs indicate a more marked anemia inducing a deficiency of oxygenation of the brain. These signs include pulsating headaches; tinnitus or tinnitus phosphenes or "flying flies" or "butterflies" in front of the eyes; false vertigo, especially when going to standing or even lipothymies. Asthenia or fatigue is later, although it is frequently reported, because the most common cause of anemia is a profound deficiency of iron. But iron is also involved in the myoglobin which is the basic protein of the muscles with fatigability when walking. Iron also intervenes in certain cytochromes, hence this frequent and early asthenia in so-called iron deficiency iron deficiency anemia's. When the anemia is very severe, a coma can occur causing death (Stockman,1986), (Kumar, Abbas, \& Aster, 2017).

Anemia can also aggravate pre-existing cardiovascular conditions: decomposition of heart failure or arthritis of the lower limbs. Finally, if the anemia is deep and brutal, it can induce a type of angina pectoris that can lead the patient to cardiac resuscitation. This mimics a crisis of angina or an infarction but in this case the coronary arteries are radiologically normal. Anemia can of course also decompensate for pre-existing coronary insufficiency. At cardiac auscultation, a functional murmur (i.e., with no underlying cardiac abnormality) can be found. Indeed, the fall in the number of red blood cells in the blood causes a drop in blood viscosity. The normally laminar flow in the heart becomes turbulent and therefore creates a reversible heart murmur after correction of anemia (Ludwig \& Strasser, 2001), (Qaseem, Humphrey, Fitterman, Starkey\& Shekelle, 2013).

Tolerance of anemia, from which the indication of blood transfusions results, depends on several factors: the level of anemia, the degree of hemoglobin decrease, the rate of hemoglobin decline (chronic anemia is much better tolerated than 'acute anemia), and the presence of other diseases, especially cardiovascular (limit tolerance). Hemoglobinconcentration mightin some casesgo down to $3 \mathrm{~g} / \mathrm{dll}$ which is such case will risk life and need immediate medical interference, as the threshold of tolerance is $>7 \mathrm{~g} / \mathrm{dl}$, (Karkouti, Wijeysundera, Yau, McCluskey, Van Rensburg, \& Beattie, 2008), (Hare, 2014).

\subsection{Anemia Development Mechanisms}

There are three main mechanisms of anemia development;1) Anemia as a consequence of disruption of normal erythrocyte formation and hemoglobin synthesis. Such a mechanism of development is observed in the case of a lack of iron, vitamin B12, folic acid, and during diseases of the red bone marrow. Sometimes anemia occurs when taking large doses of vitamin C (vitamin C intake in large doses blocks the action of vitamin B12, necessary for hematopoiesis).2) Anemia as a consequence of red blood cellsloss that is mainly due to acute bleeding (trauma, surgery). It should be noted that with chronic bleeding of small volume, the cause of anemia is not so much the loss of red blood cells, butthe lack of iron that develops against a background of chronic blood loss.3) Anemia as a result of accelerated destruction of red blood cells. Normally, the life span of red blood cells is about 120 days. In some cases (hemolytic anemia, hemoglobinopathies, etc.), erythrocytes are destroyed more quickly, which causes anemia. In many cases the destruction of red blood cells is accelerated by the use of significant amounts of vinegar, which causes the accelerated decay of red blood cells(Ishani, Weinhandl, Zhao, Gilbertson, Collins, Yusuf, \& Herzog, 2005), ( Zeuzem, DeMasi, Baldini, Coate, Luo, Mrus \& Witek, 2014). 


\subsection{Clinical Manifestations}

Often, anemia occurs without pronounced manifestations and often remains unnoticed, in many cases it is discovered by an accidental laboratory test withoutany specific complaints. Those who are suffering from anemia might notice some manifestations due to the development of anemic hypoxia. In mild forms, it can be weakness, fatigue, general malaise, and a decrease in concentration of attention. People with more severe anemia can complain of shortness of breath with mild or moderate exercise, palpitations, headache, tinnitus, sleep, appetite, and sexual desire may also occur. With very severe anemia, or with the presence of concomitant pathology, it is possible to develop heart failure (Ludwig \& Strasser, 2001), (Qaseem, et.al., 2013), (Kumar, Abbas, \& Aster, 2017).

A frequently encountered diagnostic symptom of moderate or severe anemia is pallor (skin, visible mucous membranes and nail lodges). Also and important,are symptoms such as the development of cheilosis and coilonichia, increased heart beat and the emergence of functional systolic noise.Manifestations of acute and severe anemia are always more pronounced than chronic and moderate severity.In addition to general symptoms directly associated with hypoxia, anemia may have other manifestations depending on their etiology and pathogenesis. For example, the development of sensitivity disorders with B12-deficiency anemia, jaundice - with hemolytic anemia, etc.(Rodak, Fritsma, \& Doig, 2007), (Kumar, Abbas, \& Aster, 2017).

\subsection{Anemia in Pregnancy}

Of all types of anemia in pregnancy, iron deficiency anemia most often occurs. This is due to the increasing need of iron from 0.6 to $3.5 \mathrm{mg}$ / day, which exceeds the ability to absorb it from food (1.8-2 mg / day). Iron is used to form the fetus and the placenta.If the disease persists with pregnancy, it can lead to serious consequences:the fetus may lose oxygen, which is necessary for normal development, especially the brain;women with severe forms of anemia feel worse during pregnancy; the probability of premature birth is growing;after birth, the risk of developing infections(Rodak, Bernadette 2007).

\subsection{Digital Image Processing in Anemia Diagnosis}

Digital image processing is clinically pertinent in its application to microscopic histopathological images. Thus, in a field where traditionally depends on the qualified eye of a pathologist to make diagnoses from a qualitative perspective, computer automation and diagnosis is now possible with digital images. Itdevelops algorithmic and automated methods toprovidea reliable, efficient and powerful tool which aids in data collection, assist researchers to further their understanding of the condition, and ultimately help with the diagnosis of anemia (Chard, 1990), (Goldfarb, 2001). Early identification of anemia is particularly important because most such diseases are readily treatable if diagnosed at early stages to save patient life (Barpanda, 2013).Current screening methods usually involve visual inspection of suspicious blood smear slides. According to the Centers for Disease Control (CDC, 2016), anemia and thalassemia are very common in people from Asia, Middle East, Africa, and Mediterranean countries such as Greece and Turkey (Kotila, 2012).

The objective of this paper is to develop an automated approach for the diagnosis of different anemia types using image processing. It introduces an automated method for diagnosis of blood smear microscopic images for different types of anemia and this method is tested on samples consisted of 500 microscopic images. The proposed method's starts with the laboratory preparation and ends with the diagnosis of blood smear image whether it contains anemia or not and the type of anemia. After the laboratory preparation is done, the image is preprocessed to enhance them and remove the noise. Then, color based segmentation is applied, after that, the anemia is diagnosed using bag of features and each type of the anemia is determined.

In order to achieve this paper objectives, the following steps and procedures were performed; firstly, was to investigate and study previous methods and researches that have been done in the field of analyzing microscopic histology images in order to determine the field that needs more researches and investigations. Secondly,data was collected from previous patient record of 500 patients, a sample of 500 digital images for both non anemia and anemia cases have been studied. And thirdly, a new algorithm was developed to process and automatically diagnose microscopic image of different anemia types. Fourthly, an implementation of the algorithm was done using Matlab image processing toolbox, and finally, testing the implemented system with all samples that was selected in step one, storing the results and analyzing them.

The rest of this paper is organized as follows. In Section2, related work is presented, Section 3 describes the proposed approach, Section 4 provides theexperimental results obtained by implementing the proposed approach. Finally, Section 5 contains the conclusion and future work.

\section{Related Work}

Image processing plays a vital role in the study and analysis of erythrocytes (Gonzalez, 2007). Blood disorders 
can be classified based on the comparison of feature related to shape, area, perimeter, diameter, deviation, area proportion, target flag, central pallor etc...(Taherisadr, Nasirzonouzi, Baradaran, Mehdizade \& Shiraz,2013). Other type of classification can be done using segmentation, feature extraction using artificial neural network. Classification of cells is done based on image features like texture, color and the cell geometry (Veluchamy, Perumal, \& Ponuchamy2012).

Over the decades, many researchers have been workingon finding solutions to the problem of segmentation. A detailed survey can be found in (Meijering,2012). There are numbers of segmentation techniques such as Snakes or Active Shape Models (Bengtsson, Wahlby \& Lindblad 2004), (Park and Keller, 2001), (Nguyen, Worring, \& Van Den Boomgaard, 2003). Makkapati and Rao(2009) and Savkare and Narote(2012) used median filter for noise reduction and smoothening. In median filter, all pixel values are placed in an ascending order and median value is kept at the center of filter.

Digital image are used to automatically analyze erythrocytes using image processing techniques using the Matlab (Gonzalez, Woods, \& Eddins, 2009). Previous study gives information about evolution of the gene that produces sickle shaped hemoglobin (Chard, 1990). Other research focuses on how natural selection of human gene can provide increased adaptive fitness when exposed to an infectious disease (Chen, \& Rajewsky, 2006).

There is a new technique for the shape analysis of any fractal object using fractal dimension techniques. This is mostly used when it is required to detect manmade object from an image (Sarkar, \& Chaudhuri, 1994). Local fractal dimension can be used to discriminate among object in gray scale imagery (Lopes, \& Betrouni, 2009). The box counting algorithm for computing local fractal dimension promises real time result (Collett, Despland, Simpson, \& Krakauer, 1998), (Chen, Lee, Han, Shih, \& Chang, 2013). This method show ambiguities in the mass scaling technique based on shape and range. This shows basic idea or using fractal dimension as an important technique for object identification which can be used with image processing.

Microscopic histopathology image processing may be done for all the magnifications of the microscope $(\times 2, \times 4.5$, $\times 10, \times 20$, and $\times 40$ ) for diagnosis and classification (Gurcan, Boucheron, Can, Madabhushi, Rajpoot, \& Yener, 2009). It may be at lower magnification for microscopic tissue level analysis. As Demir, \& Yener, (2005) explained for both tissue level and cell level analysis methods for cancer diagnosis. They analyzed histopathology tissue images using image preprocessing, feature extraction and classification techniques such as morphological processing, threshold, boundary based, region based, and classification methods.

With the histopathology digital images based on likelihood function estimation; cancer cells have been detected with $81.1 \%$ sensitivity based on component wise threshold. Many analysis ways may also applied to breast cancer histopathology images (Veta, Van Diest, Kornegoor, Huisman, Viergever, M. \& Pluim, 2013). Basedon the system or type of illness, the digital image analysis steps may be different.

Rakshit, and Bhowmik, (2013) have used metric values to differentiate normal RBCs from sickle cells with an accuracy of $95.8 \%$. Bala and Doegar, (2015) have focused on de-segmentation ofoverlapping cells using wateritd segmentation to improve accuracy of detection. Veluchamy, Perumal, \& Ponuchamy, (2012)have focused on feature extraction and classification of blood cells using artificial neural network.Sahu,Biswas, \& Uma(2015) have usedvarious segmentation methods such as edge detectionalgorithm, cluster based segmentation, thresholding, fractaldimension and box counting to increase the accuracy ofdetection.

Savkare, \& Narote, (2015) have proposed automated malaria detection devices using digital image processing for fast diagnosis and acute treatment of Malaria. In 2014 Bashir, Qamar, Khan andJaved devised rule based methods for classification of diabetes using ID3, C4.5 and CART ensembles. In 2009 Zhang, Zeng, Wu, Zhang and Jiang classified breast cancer metastasis usingEnsemble Learning Techniques. In 2014 Zhang, Wu and Hu classified text using Ensemble Learning Methods.In 2013 Lan, and Gaoused neural network to combine multiple classifiers. In 2012 Wang, Gao, Wang and Miu used C5.0 decision tree of multiple combined classifiers to devise a new classification method. In 2012 Veluchamy, Perumal and Ponuchamy used 4 geometrical features, 16 statistical features and 7 moment invariant features to train back propagation neural network to classify the blood cells with classification efficiency of $80 \%$ and $66.6 \%$ for normal and abnormal cells respectively. In 2008 Poomcokrak and Neatpisarnvanit used MLP (Multilayer perceptron) to classify normal RBCs and Sickle cells.

\subsection{Overview for the Automation of Histological Image Analysis}

Traditionally, histology has been considered as a descriptive science, but recently it is actively changing, which is mainly due to the use of methods of quantitative analysis. Such transformations allow speaking about quantitative histology. Using digital image analysis we can measure the quantity of certain features in the tissue or blood smear and determine the disease (Johnson, Del Brady, Fuhrman, Abdul-Karim, O. A., \& Shah, 2006), (Gurcan, et.al., 
2009). Researches of this kind are added to the study of pathological processes at the tissue level, and the discipline itself is called pathohistology. Automation the analysis of histopathology images have been a very important research subject with the revolution of computer and image processing development. An overview for the automation of histological image processing is shown in Figure 1.Quantitative analysis using image processing makes it possible to more effectively find the relationships between the structure and function of tissues and / or cells, improve the accuracy of the estimates obtained, reduce the influence of the subjective factor on the results of the analysis, and automate the research and diagnostic procedures (Sutton, 2005), (Belsare, \& Mushrif, 2012).

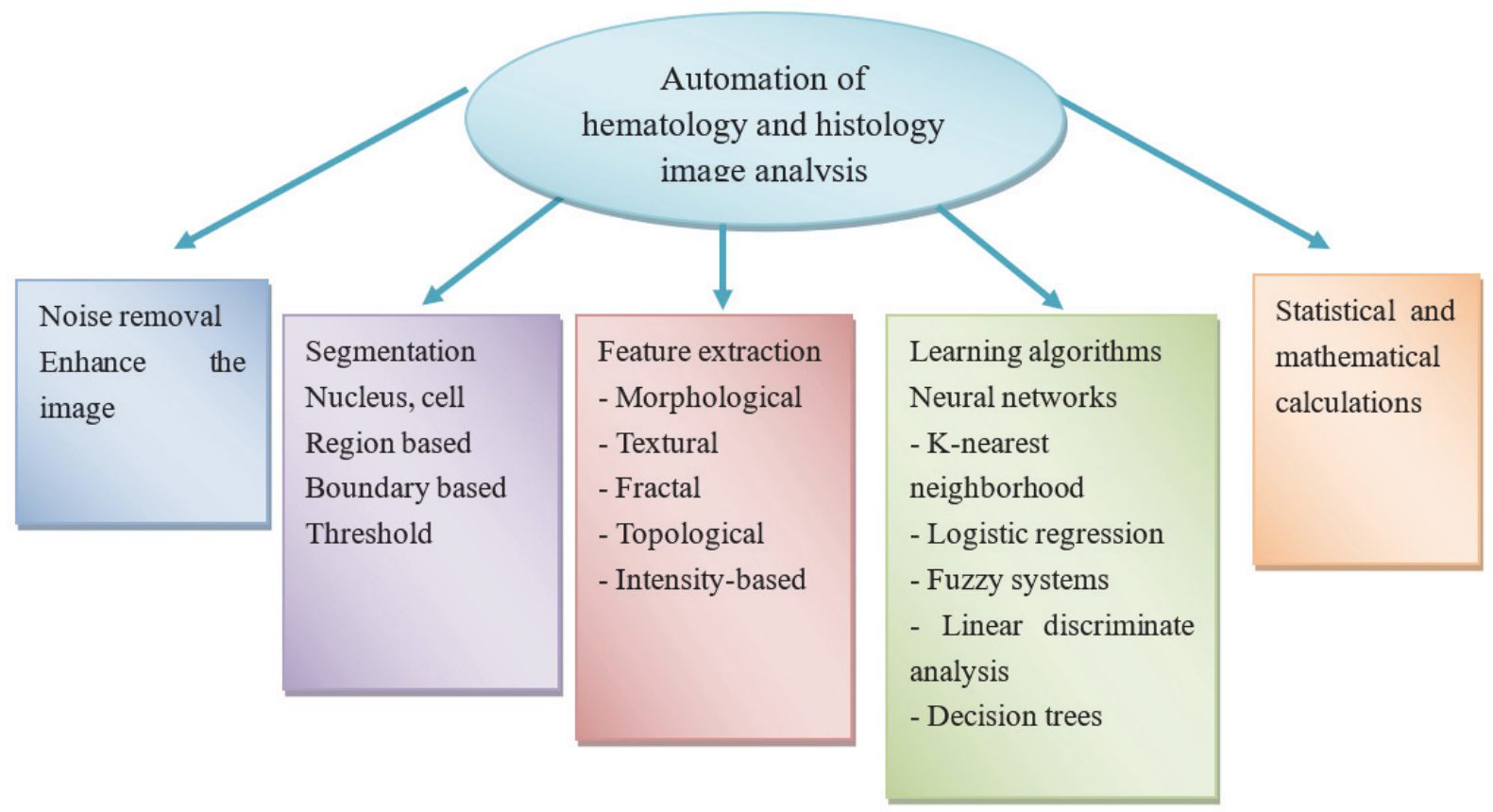

Figure 1. Overview of Automation of hematology and histology image analysis

\section{Proposed Methodology}

We propose an automated approach for anemia diagnosis, it uses colored-based images to diagnose anemia as shown in figure 2, the proposed Image analysis and recognition include the phases of Image preprocessing and detection of iron deficiency Anemia cells see figure 3.

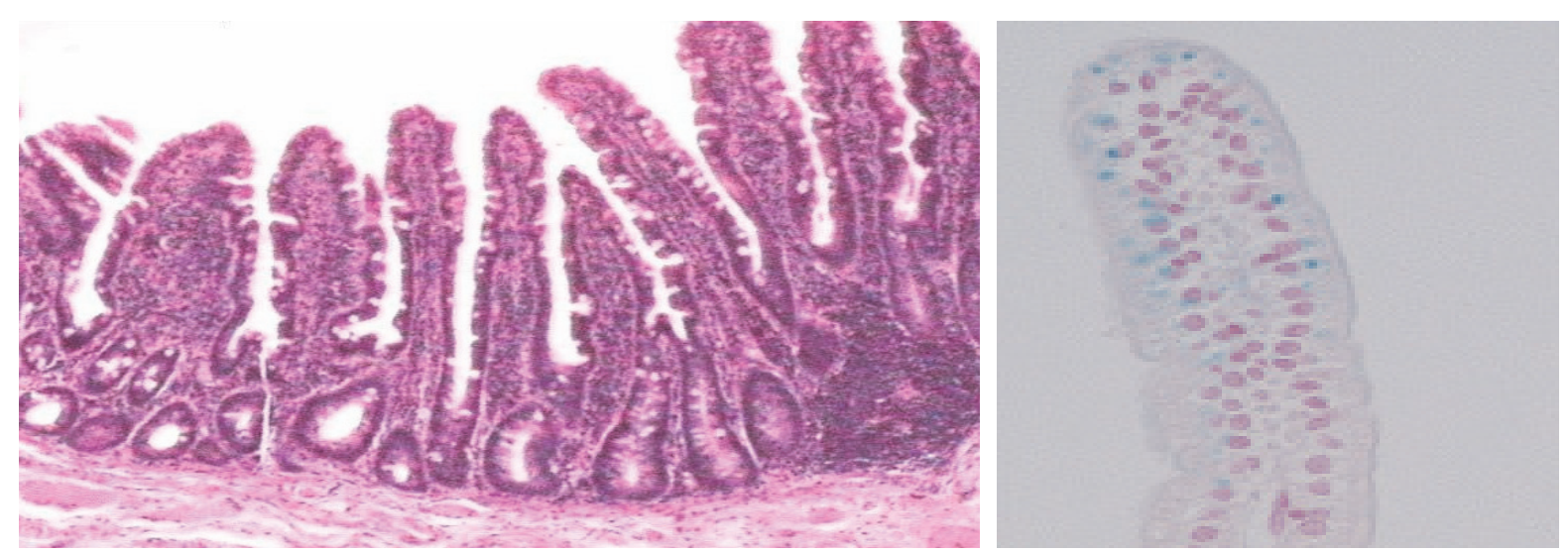

Figure 2. Section of Small intestine image that contains intestine villi 


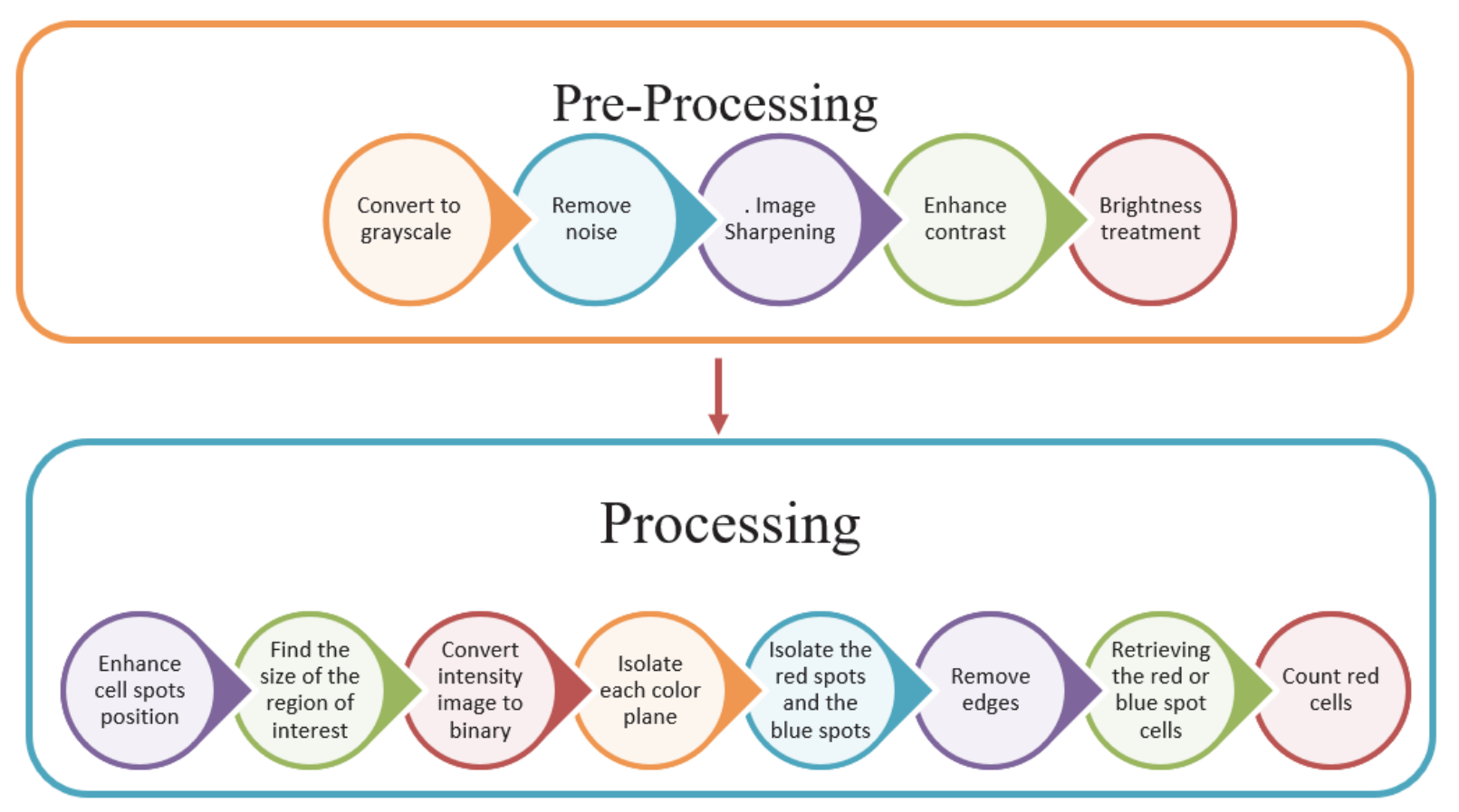

Figure 3. Main Phases of the proposed methodology

\subsection{Preprocessing Phase}

The preprocessing is an essential phase in microscopic histology image analysis because of the nature and circumstances of the slides preparation, staining and image shooting, which affect the quality of the image. The preprocessing phase aims at enhancing the image by performing the five steps shown in figure 4afterImage acquisitioning. After applying the preprocessing phase the analysis continues to the processing phase that leads to the detection and diagnosis of anemia in the image.

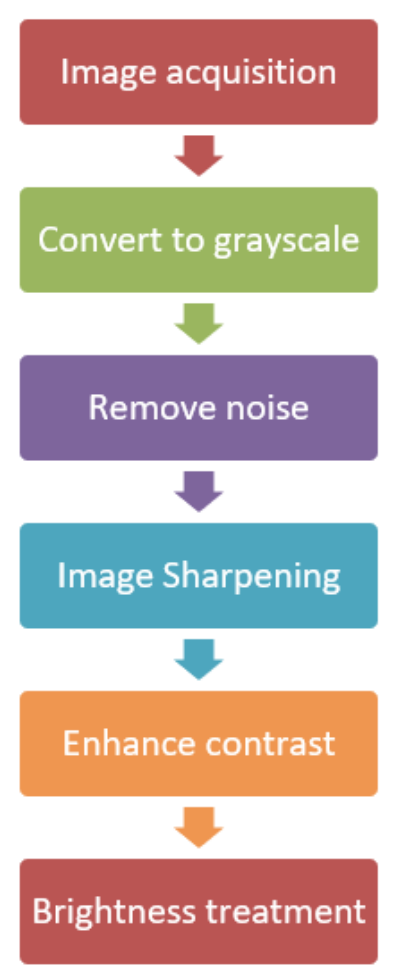

Figure 4. Preprocessing Phase Steps 


\subsubsection{Convert Image to Grayscale}

The first step is converting the selected image that consists of $\mathrm{x}$ red spot blood stained cells and B blue spot blood stained cells that is located in the intestine villi as shown in figure 5. Converting the image from RGB images to grayscale by eliminating the hue and saturation information while retaining the Luminanceand this can be done first by applying stretching transformation and that's in order to increase the concentration of the image and will make all the image colors used from (0 to 255). Convert image from (RGB) to grayscale mainly aims to enhance cells position color plane at the pixel's location with 8 bits for each color, giving a potential of 16 million colors and make it difficult to determine the position of the spot using all this combination, so we need to convert it to gray scale image. The gray scale image values range from $x \min$ to, $x \max$ e.g. The gray value of the image at point $\mathrm{x}=(\mathrm{X}, \mathrm{Y})$, the lighter the gray value at point $\mathrm{x}$, the higher the altitude of the corresponding point $\{\mathrm{x}, \mathrm{f}\}$ on the surface of the image. So the lower points and the zero values may represent the points the spaces between cells like the background points and the points that contain noise; if the value of the function $f=0$ then this point may represent the cells and it location is at a pixel $(\mathrm{X}, \mathrm{Y})$ in which the value of each pixel is a single sample, that is, it carries only intensity information. Grayscale images are often the result of measuring the intensity of light at each pixel in a single band of the electromagnetic spectrum and this will represent the existence of cells or nuclei in the studied image

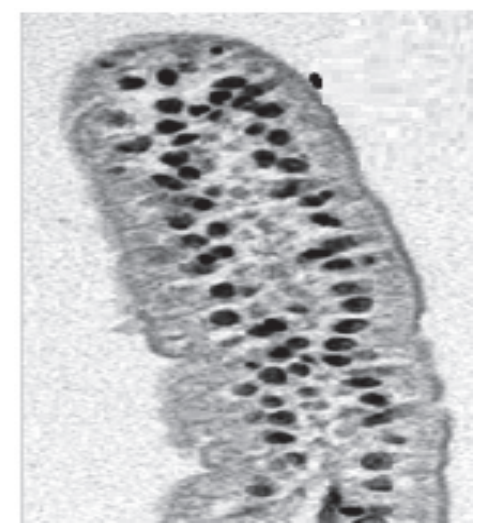

Figure 5. Section of Small intestine image that contains intestine villi after applying the Convert image to grayscale step

\subsubsection{Noise Removal}

Noise removal is done by applying fuzzy filter for the colored image to remove noise as shown in figure 6.Noise in microscopic images can occur during image transmission, capture or preparation of the slide images. That's why we need to remove the noise and enhance the image, we have applied fuzzy filter to remove the noise, applying fuzzy filters for noise removal gives great results in many digital image analyses such as microscopic image which overcome some malfunctions of classical filters. In the situation of the microscopic histology images fuzzy filter is very helpful for removing noise. Fuzzy filter that takes the nearest data to remove the noise, it also performs edge preservation.

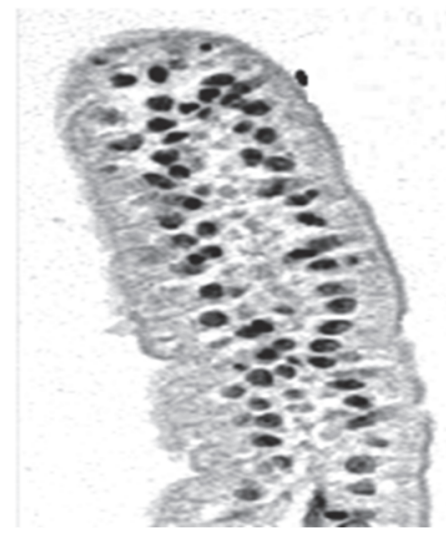

Figure 6. Section of Small intestine image that contains intestine villi after applying Noise Removal step 


\subsubsection{Image Sharpening}

Because of the fact that all camera's produce soft images as though digital image for the microscopic Histopathological tissue are soft so there is a need to sharpening the image and to do this we have applied the shock filter as shown in figure 7. Shock filter is used because it is based in the idea to apply locally either dilation or erosion process, depending on whether the pixel be-longs to the influence zone of a maximum or a minimum and this is the case in the anemia cells. The decision between dilation and erosion were made using the sign functions in set $\{-1,0,+1\}$ based on the Laplace operator (Kramer-Bruckner, 1975). Applying this filter have enhanced the image and produced a sharp discontinuity called shock at the borderline between two the objects (cells and nuclei) and the background.

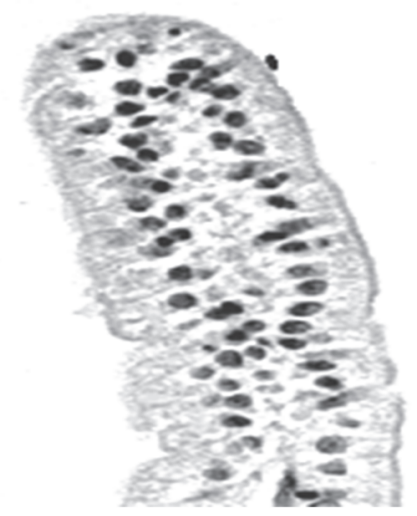

Figure 7. Section of Small intestine image that contains intestine villi after sharpening the image

\subsubsection{Enhancecontrast}

Before proceeding further to the preprocessing phase, we need to adjust image intensities to enhance contrast of the images. This step is performed by applying histogram equalization to the image which computes the probabilities per pixel and further spreads them over the whole band of $[0,255]$ to obtain Enhance contrast as shown in figure 8 .

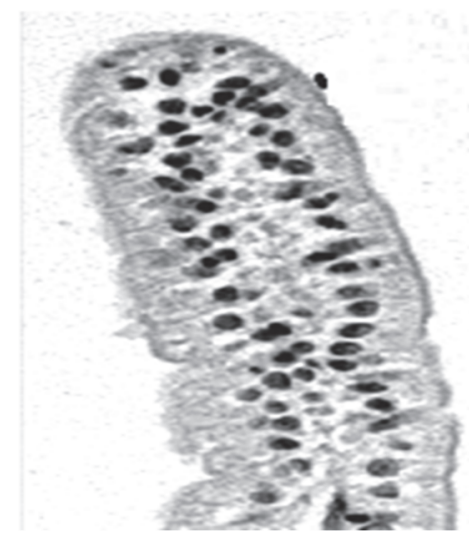

Figure 8. Section of Small intestine image that contains intestine villi after sharpening the image

\subsubsection{Brightness Treatment}

As proposed by Yi-Ming Chen, Tsao, \& Tsai, (2016)the brightness treatment is essential step in anemia diagnosis because the same camera that is used to capture palpebral conjunctiva images with the automatic white balance activated, some obvious bright spots appear in the original images even under just ambient light and noflash light. The bright spots are considered as a noise source because they interfere the extraction of reliable recognition features. It is understood that not all images were taken at the same time or at the same ambient light conditions. Therefore, the bright spots may have different shapes and sizes from one image to another. They must be handled well in order to reveal more reliable characteristics for recognition. We use K-means clustering to identify the bright spot area in the gray-level image converted from a corresponding RGB color image as shown in figure 9 


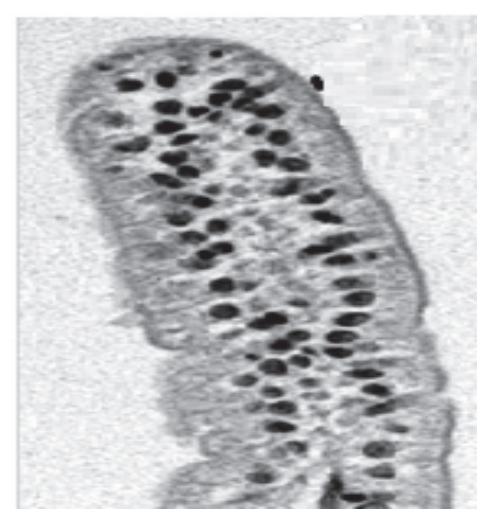

Figure 9. Section of Small intestine image that contains intestine villi after Brightness treatment step

\subsection{Processing and Detection Phase}

We presented in this work a new methodology for detecting anemia as shown in figure 10that describes the steps of the processing phase. The first step is enhancing cell spots position, fined the size of the region of interest (ROI), convert intensity image to binary, isolate each color plane alone, isolate the red spots and the blue spots and remove the edges, retrieving the red or blue spot cells and finally Count red cells
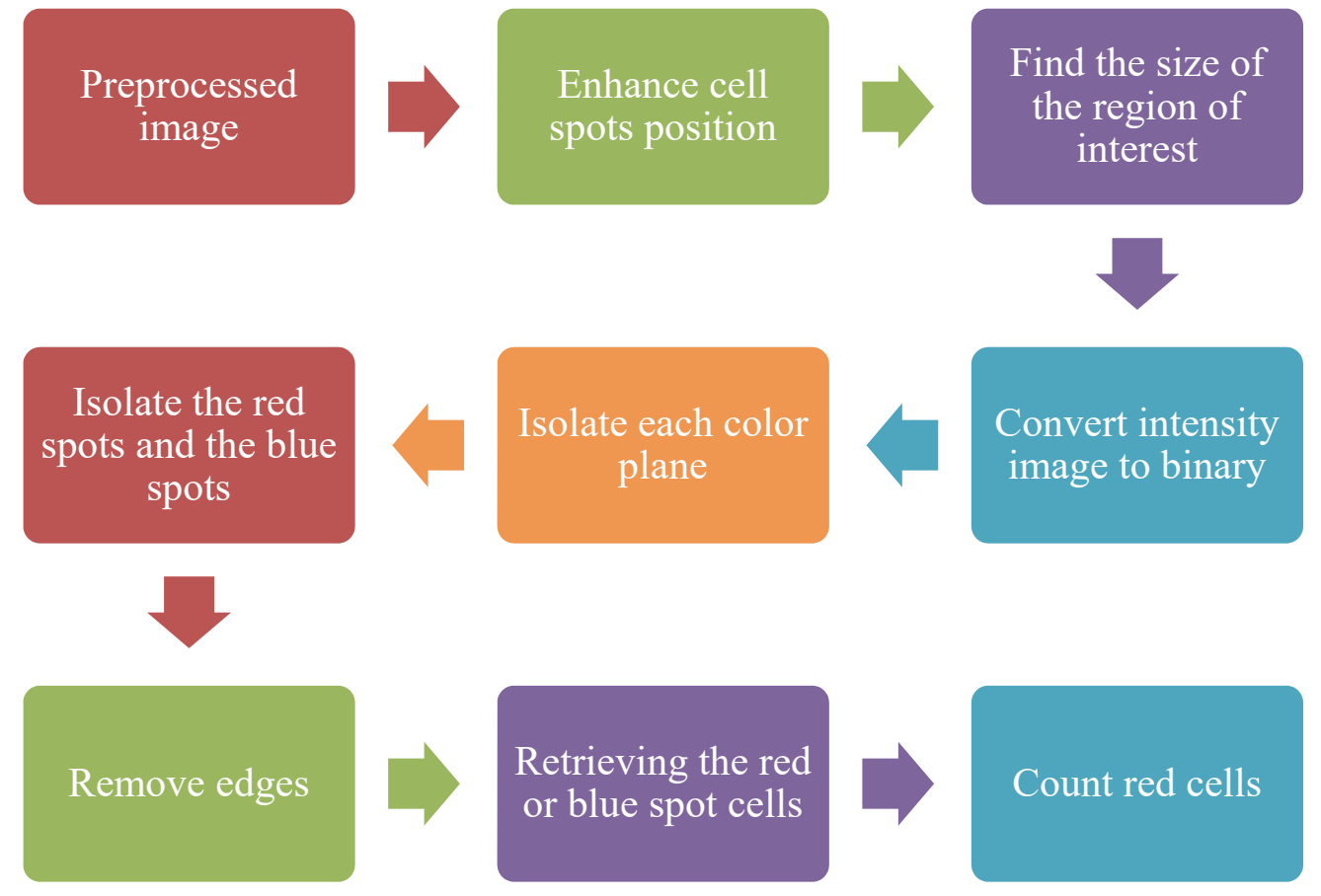

Convert intensity

image to binary

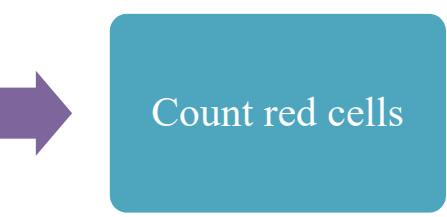

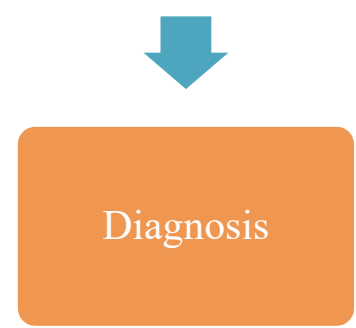

Figure 10. Processing and detection phase steps

\subsubsection{Enhance Cell Spots Position}

This step mainly aims to enhance cell spots position determination since each pixel in the RGB image is determined 
by the combination of the red, green, and blue intensities that is stored in each color plane at the pixel's location with 8 bits for each color, giving a potential of 16 million colors and make it difficult to determine the position of the cell spots using all this combination; so we need to convert it to gray scale image.

\subsubsection{Find the Size of the Region of Interest (ROI)}

This step aims at counting the number of cells in or the hall image as determined by the analyst, the region of interest represent a complete villi that contains cells, because in some images we will notice part of villi not a complete one as resulted from the cut of the intestine in preparation time as shown in figure11.

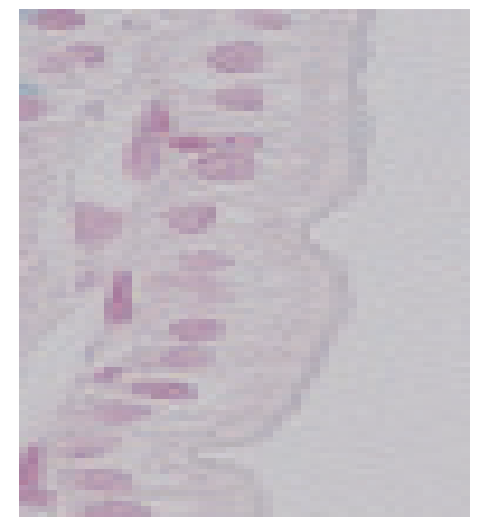

Figure 11. Section of Small intestine image that contains intestine villi after Find the size of the region of interest step

\subsubsection{Isolate Each Color Plane Alone}

Isolate each color plane alone is applied to the original stretched binary image not the grayscale image that is resulted in the previous step and it includes creating new image by using threshold. Computing an appropriate threshold to use it to convert the intensity image to binary and in binary image, each pixel assumes one of only two discrete values. Essentially, these two values correspond to on and off. A binary image is stored as a logical array of 0's (off pixels) and 1's (on pixels). The output binary image has values of 0 (black) for all pixels in the input image with luminance less than level and 1 (white) for all other pixels which here these pixels represent the red blood cells in the villi and the edge of the villi which will be removed in the next steps (Goldfarb, 2001), (Gonzalez, \& Woods, 2006))since the RGB image consist of green and blue and red and the green represent both red and blue colors we will isolate each color plane alone and this will give us 2 new images as show in Figure 12 and Figure 13.

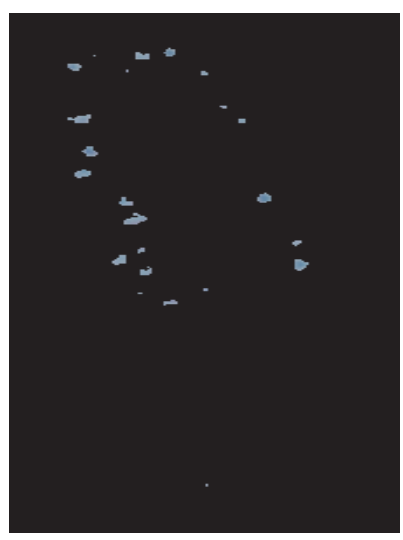

Figure 12. Section of Small intestine image that contains intestine villi after Isolate green color plane alone step

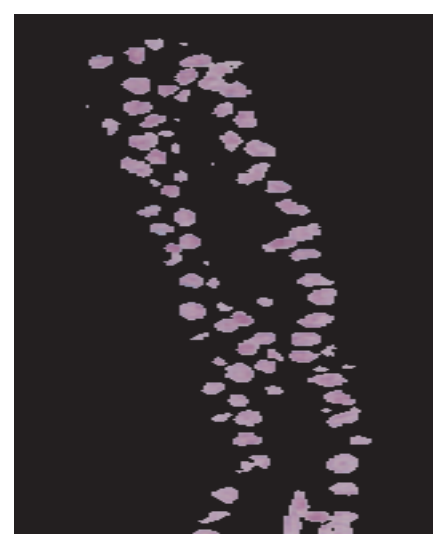

Figure 13. Section of Small intestine image that contains intestine villi after Isolate redcolor plane alone step

\subsubsection{Isolating the Red Spots and the Blue Spots and Removing the Edges}

This step proposed an algorithm to isolate the red spots and the blue spots from the background after that we 
remove the edges that have resulted from the first step because these edge will give errors while continuing to do this dilation which was applied to the image so that it will remove the edges (Gonzalez, \& Woods, 2006).

\subsubsection{Retrieving the Red or Blue Spot Cells}

Retrieving the red or blue spot cells were eliminated by the isolation of the red, blue and background new produced sub images by intersecting the images together and this will make some of the lost spots appear as shown in Figure 14, Figure 15.

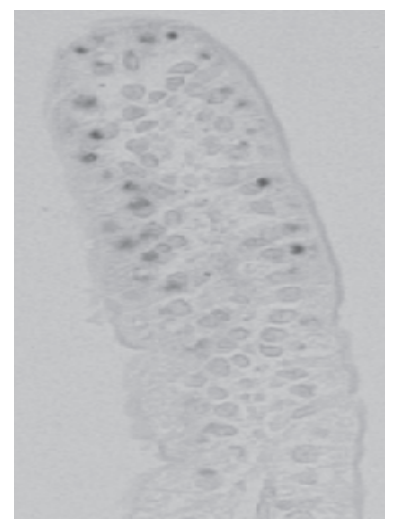

Figure 14. Retrieving the blue spot cells from Section of Small intestine image that contains intestine villi

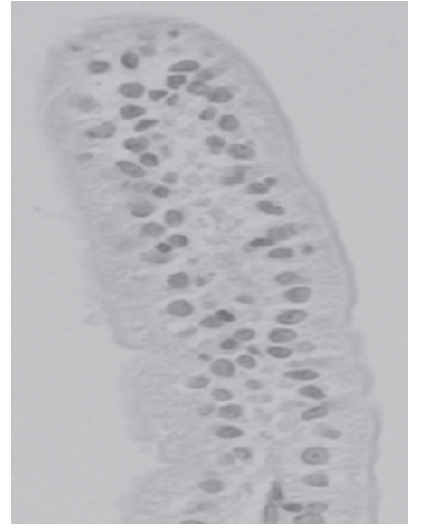

Figure 15. Retrieving the red or blue spot cells from Section of Small intestine image that contains intestine villi

\subsubsection{Determining the Spots Location}

The next step is to determine the number of blue and red spot cells that is found in the ROI in the binary image after removing the noise from the image, and this is done first by locating the place where the spots are found and this is done first by applying dilation as shown in Figure 16, Figure 17to the blue sub image that is resulted from the previous step and then to the red plane sub image after that the spots number is calculated (Matlab, 2007)

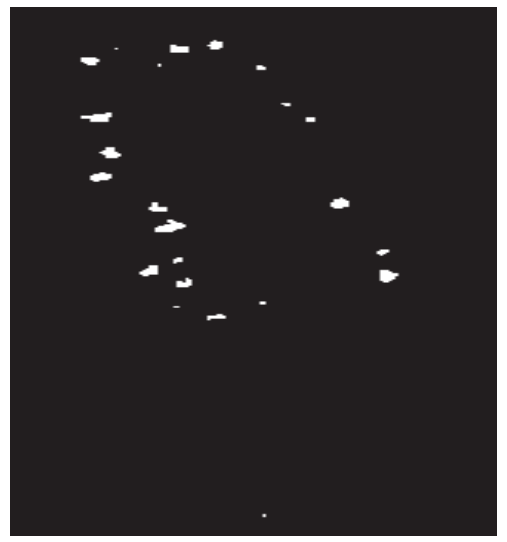

Figure 16. determining the locating of the blue cells by applying dilation in a Section of Small intestine image that contains intestine villi

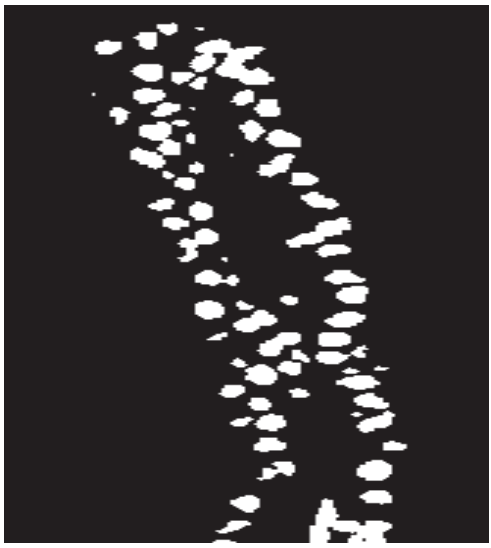

Figure 17. determining the locating of the red cells byapplying dilation in a Section of Small intestine image that contains intestine villi

\subsubsection{Count Number of Red Cells}

The final step is displaying the results that is needed in a fast and clear way for analysis and caparison studied and for continued researches the results will be displayed for the analyst showing the number of red cells, the number of blue cells and the percentage between them. The proposed approach depends on the automation of the counting process of blue and red cells in the studied image. Further, gives the analyst a better control over the counting process and he can choose any part of the image or the whole image to study and fast count of the cell spots. 


\section{Experimental Results and Implementation}

The experimental results of the methodology were carried out in a set of 100 image of small intestine that contain intestine villi, obtained from Grand Medical Laboratory (GML) in Jordan.

The conventional method of testing sickle cell anemia is by forming a thin blood smear and observing it under microscope. Thin blood smear can be used to efficaciously detect the presence of sickle cells on the basis of visual criteria. Considering this the microscopic images are acquired from reliable sources such as CDC, etc

The images were prepared in the lab by first fixing them using Para formaldehyde to preserve the tissues then they were mounted on paraffin blocks and sliced for about approximately $2 \mathrm{~mm}$ with a microtome device. And the tissue is now put in a slide then the slides stained for iron using Gomori's Method (Gomori, 1939), (Prophet, 1992), (Luna, 1968), (Ruifrok, 1997) Nikon -Eclipse microscope with Plan Flour lenses was used and Spot Insight color camera was took the images for slides and stored to be analyzed using Matlab softwarethat were used to implement the proposed approach because Matlab is a high-performance language for education and research ,it integrates computation, visualization, and programming in an easy-to-use environment where problems and solutions are expressed in familiar mathematical notation and also it has toolboxes for signal processing, neural network, image processing, database ... etc , Matlab Image Processing Toolbox is a collection of functions that extend the capability of the Matlab numeric computing environment. The toolbox supports a wide range of image processing operations, such as Image analysis and enhancement, Region of interest operations, Linear filtering and filter design (Matlab 7, 2004).

The experimental results have shown that using digital image processing techniques through processing the image into different stages as including noise removal, image sharpening, enhancing contrast, find region of interest,isolating color, removing edges, and counting cells leads to a successful outcome and the diagnose of anemia.

\section{Conclusion and Future Work}

This paper described a new automated approach for determining spot number of red cells and the number of blue cells that represent the iron deficiency in the cell and also calculate the ratio between them in order to determine the percentage of the presence of blue cells compared to the hall number of cells it improves cell number determination by making it automated, also because it is very fast method that require seconds for analyzing the image and get the result

Iron deficiency cell image analysis is a very interesting research area that's why our future work intended to pursue the research for developing a new algorithm for automation all types of Iron deficiency image that is done manually in laboratory and take a lot of time.

\section{References}

Bala, S., \& Doegar, A. (2015). Automatic detection of sickle cell in red blood cell using watershed segmentation. Int. J. Adv. Res. Comput. and Commun. Eng, 4(6), 488-491.

Barpanda, S. S. (2013). Use of Image Processing Techniques to Automatically Diagnose Sickle-Cell Anemia Present in Red Blood Cells Smear (Doctoral dissertation).

Bashir, S., Qamar, U., Khan, F. H., \& Javed, M. Y. (2014, December). An Efficient Rule-Based Classification of Diabetes Using ID3, C4. 5, \& CART Ensembles. In 2014 12th International Conference on Frontiers of Information Technology (FIT) (pp. 226-231). IEEE.

Belsare, A. D., \& Mushrif, M. M. (2012). Histopathological image analysis using image processing techniques: An overview. Signal \& Image Processing, 3(4), 23.

Bengtsson, E., Wahlby, C., \& Lindblad, J. (2004). Robust cell image segmentation methods. Pattern Recognition and Image Analysis C/c of Raspoznavaniye Obrazov i Analiz Izobrazhenii., 14(2), 157-167.

Beutler, E., \& Waalen, J. (2006). The definition of anemia: what is the lower limit of normal of the blood hemoglobin concentration? Blood, 107(5), 1747-1750.

Beutler, E., \& West, C. (2005). Hematologic differences between African-Americans and whites: the roles of iron deficiency and $\alpha$-thalassemia on hemoglobin levels and mean corpuscular volume. Blood, 106(2), 740-745.

Calbet, J. A., Lundby, C., Koskolou, M., \& Boushel, R. (2006). Importance of hemoglobin concentration to exercise: acute manipulations. Respiratory Physiology \& Neurobiology, 151(2-3), 132-140.

Centers for Disease Control
https://www.cdc.gov/immigrantrefugeehealth/guidelines/domestic/general/discussion/complete-blood- 
count.html

Chard, T. (1990). Laboratory Techniques in Biochemistry and Molecular Biology. El Sevier, Amsterdam-New York-Oxford: 216-219.

Chen, H. M., Tsao, Y. T., \& Tsai, S. C. (2016). Automatic Image Segmentation Scheme for Counting the Blood Cell Nuclei with Megaloblastic Anemia. Journal of Medical Imaging and Health Informatics, 6(1), 102-107.

Chen, K., \& Rajewsky, N. (2006). Natural selection on human microRNA binding sites inferred from SNP data. Nature genetics, 38(12), 1452.

Chen, W. K., Lee, J. C., Han, W. Y., Shih, C. K., \& Chang, K. C. (2013). Iris recognition based on bidimensional empirical mode decomposition and fractal dimension. Information Sciences, 221, 439-451.

Collett, M., Despland, E., Simpson, S. J., \& Krakauer, D. C. (1998). Spatial scales of desert locust gregarization. Proceedings of the National Academy of Sciences, 95(22), 13052-13055.

Demir, C., \& Yener, B. (2005). Automated cancer diagnosis based on histopathological images: a systematic survey. Rensselaer Polytechnic Institute, Tech. Rep.

Goldfarb, M. F. (2001). Analysis of casein using two-dimensional electrophoresis, western blot, and computer imaging. Adv. in Exp Med and Biol., 501, 535-539.

Gomori, G. (1939). A differential stain for cell types in the pancreatic islets. The American Journal of Pathology, 15(4), 497.

Gonzalez, R. C., \& Woods, R. E. (2006). Digital Image Processing (3rd Ed). Prentice-Hall, Inc., Upper Saddle River, NJ, USA.

Gonzalez, R. C., Woods, R. E., \& Eddins, S. L. (2009). Digital Image processing using MATLAB®. United States: Gatesmark Publishing.

Gurcan, M. N., Boucheron, L., Can, A., Madabhushi, A., Rajpoot, N., \& Yener, B. (2009). Histopathological image analysis: A review. IEEE Reviews in Biomedical Engineering, 2, 147.

Hare, G. M. (2014). Tolerance of anemia: understanding the adaptive physiological mechanisms which promote survival. Transfusion and Apheresis Science, 50(1), 10-12.

Ishani, A., Weinhandl, E., Zhao, Z., Gilbertson, D. T., Collins, A. J., Yusuf, S., \& Herzog, C. A. (2005). Angiotensin-converting enzyme inhibitor as a risk factor for the development of anemia, and the impact of incident anemia on mortality in patients with left ventricular dysfunction. Journal of the American College of Cardiology, 45(3), 391-399.

Janz, T. G., Johnson, R. L., \& Rubenstein, S. D. (2013). Anemia in the emergency department: evaluation and treatment. Emergency medicine practice, 15(11), 1-15.

Johnson, P. C., Del Brady, M., Fuhrman, M. G., Abdul-Karim, O. A., \& Shah, S. (2006).Method for quantitative analysis of blood vessel structure U.S. Patent No. 6,993,170. Washington, DC: U.S. Patent and Trademark Office.

Karkouti, K., Wijeysundera, D. N., Yau, T. M., McCluskey, S. A., Van Rensburg, A., \& Beattie, W. S. (2008). The influence of baseline hemoglobin concentration on tolerance of anemia in cardiac surgery. Transfusion, 48(4), 666-672.

Kotila, T. R. (2012).Thalassaemia is a tropical disease. Annals of Ibadan Postgraduate Medicine, 10(2), 11-15.

Kumar, V., Abbas, A. K., \& Aster, J. C. (2017). Robbins basic pathology e-book. Elsevier Health Sciences.

Lan, Y. D., \& Gao, L. (2013). A New Model of Combining Multiple Classifiers Based on Neural Network. In Emerging Intelligent Data and Web Technologies (EIDWT), 2013 Fourth International Conference on (pp. 154-159). IEEE.

Lopes, R., \& Betrouni, N. (2009). Fractal and multifractal analysis: a review. Medical Image Analysis, 13(4), 634649.

Ludwig, H., \& Strasser, K. (2001). Symptomatology of anemia. In Seminars in oncology (Vol. 28, pp. 7-14). WB Saunders.

Luna, L. G. (1968). Manual of Histological Staining Methods of the Armed Forces Institutes of Pathology. McGraw-Hill Book Company, New York-Toronto-London-Sydney:179.

Makkapati, V. V., \& Rao, R. M. (2009, April). Segmentation of malaria parasites in peripheral blood smear images. 
In Acoustics, Speech and Signal Processing, 2009. ICASSP 2009. IEEE International Conference on (pp. 1361-1364). IEEE.

Meijering, E. (2012). Cell segmentation: 50 years down the road [life sciences]. IEEE Signal Processing Magazine, 29(5), 140-145.

Nguyen, H. T., Worring, M., \& Van Den Boomgaard, R. (2003). Watersnakes: Energy-driven watershed segmentation. IEEE Transactions on Pattern Analysis and Machine Intelligence, 25(3), 330-342.

Park, J., \& Keller, J. M. (2001). Snakes on the watershed. IEEE Transactions on pattern analysis and machine intelligence, 23(10), 1201-1205.

Poomcokrak, J., \& Neatpisarnvanit, C. (2008). Red blood cells extraction and counting. In The 3rd International Symposium on Biomedical Engineering (pp. 199-203).

Prophet, E. B. (1992). Laboratory methods in histotechnology. Amer Registry of Pathology.

Qaseem, A., Humphrey, L. L., Fitterman, N., Starkey, M., \& Shekelle, P. (2013). Treatment of anemia in patients with heart disease: a clinical practice guideline from the American College of Physicians. Annals of Internal Medicine, 159(11), 770-779.

Rakshit, P., \& Bhowmik, K. (2013). Detection of abnormal findings in human RBC in diagnosing sickle cell anaemia using image processing. Procedia Technology, 10, 28-36.

Rodak, B. F., Fritsma, G. A., \& Doig, K. (2007). Hematology: clinical principles and applications. Elsevier Health Sciences.

Ruifrok, A. C. (1997). Quantification of immune histochemical staining by color translation and automated thresholding. Analytical and Quantitative Cytology and Histology, 19(2), 107-113.

Sahu, M., Biswas, A. K., \& Uma, K. (2015). Detection of Sickle Cell Anemia in Red Blood Cell: A Review. International Journal of Engineering and Applied Sciences, 2(3).

Sarkar, N., \& Chaudhuri, B. B. (1994). An efficient differential box-counting approach to compute fractal dimension of image. IEEE Transactions on systems, man, and cybernetics, 24(1), 115-120.

Savkare, S. S., \& Narote, S. P. (2012). Automatic system for classification of erythrocytes infected with malaria and identification of parasite's life stage. Procedia Technology, 6, 405-410.

Savkare, S. S., \& Narote, S. P. (2015). Automated system for malaria parasite identification. In Communication, Information \& Computing Technology (ICCICT), 2015 International Conference on (pp. 1-4). IEEE.

Smith, D. L. (2009). Anemia in the elderly. Iron disorders institute guide to anemia, 9, 96-103.

Stockman 3rd, J. A. (1986). Anemia of prematurity. Current concepts in the issue of when to transfuse. Pediatric Clinics of North America, 33(1), 111.

Sutton, C. D. (2005). Classification and regression trees, bagging, and boosting. Handbook of Statistics, 24, 303329.

Taherisadr, M., Nasirzonouzi, M., Baradaran, B., Mehdizade, A., \& Shiraz, I. (2013). New approch to red blood cell classification using morphological image processing. Shiraz E-Medical Journal, 14(1), 44-53.

Veluchamy, M., Perumal, K., \& Ponuchamy, T. (2012). Feature extraction and classification of blood cells using artificial neural network. American Journal of Applied Sciences, 9(5), 615.

Veluchamy, M., Perumal, K., \& Ponuchamy, T. (2012). Feature extraction and classification of blood cells using artificial neural network. American Journal of Applied Sciences, 9(5), 615.

Veta, M., Van Diest, P. J., Kornegoor, R., Huisman, A., Viergever, M. A., \& Pluim, J. P. (2013). Automatic nuclei segmentation in H\&E stained breast cancer histopathology images. PloS one, 8(7), e70221.

Wang, M., Gao, K., Wang, L. J., \& Miu, X. H. (2012, August). A novel hyperspectral classification method based on C5. 0 decision tree of multiple combined classifiers. In Computational and Information Sciences (ICCIS), 2012 Fourth International Conference on (pp. 373-376). IEEE.

Zeuzem, S., DeMasi, R., Baldini, A., Coate, B., Luo, D., Mrus, J., \& Witek, J. (2014). Risk factors predictive of anemia development during telaprevir plus peginterferon/ribavirin therapy in treatment-experienced patients. Journal of Hepatology, 60(6), 1112-1117.

Zhang, T., Wu, J., \& Hu, H. (2014, November). Text classification based on a novel ensemble multi-label learning method. In Systems and Informatics (ICSAI), 2014 2nd International Conference on (pp. 964-968). IEEE. 
Zhang, W., Zeng, F., Wu, X., Zhang, X., \& Jiang, R. (2009, August). A comparative study of ensemble learning approaches in the classification of breast cancer metastasis. In Bioinformatics, Systems Biology and Intelligent Computing, 2009. IJCBS'09. International Joint Conference on (pp. 242-245). IEEE.

\section{Copyrights}

Copyright for this article is retained by the author(s), with first publication rights granted to the journal.

This is an open-access article distributed under the terms and conditions of the Creative Commons Attribution license (http://creativecommons.org/licenses/by/4.0/). 